\title{
EVALUATING POLICY IMPACT OF LARGE WATER RESERVOIRS UNDER CLIMATE CHANGE
}

\author{
ALVARO BUENO BUORO ${ }^{1}$, EDUARDO CESAR COUTINHO $^{2}$ \& DANIEL SPECHT ${ }^{1}$ \\ ${ }^{1}$ IBM Research, Rio de Janeiro, Brazil \\ ${ }^{2} \mathrm{IME}$, Rio de Janeiro, Brazil
}

\begin{abstract}
We developed a methodology for modelling climate change impacts on reservoir levels and to evaluate the applicability of the model into the process of policy evaluation. We start by performing the technique of Quantile Mapping to apply bias correction in two Climate Change (CC) General Circulation Models (GCM) models (HADGEM and CNRM) based on an overlapping period of ten years. This data is used as input into a two state Markov Chain model to generate a stochastic rain generator model. We then suggest an alternative method to perform a smooth transition from the historical data to long term climate forecasting. These stochastic rainfalls are the input to a calibrated runoff model that is part of a dynamic simulation system incorporating the alternative policies used for the reservoir. This method created a realistic decision support tool, incorporating the uncertainty associated with the $\mathrm{CC}$ to evaluate management policies. We applied this methodology on the Cantareira reservoir, one of the largest drinking water systems in the world. It was concluded that the "seasonal policy" (RAC1) is more robust to maintain a constantly higher storage level in relation to the alternative. The climate change analysis indicates a steady increase in storage and overflow with time. Keywords: stochastic rainfall, policy evaluation, bias correction, climate change, decision support.
\end{abstract}

\section{INTRODUCTION}

The traditional approach for planning reservoir management decisions is based on historical records of pluviometry, assuming its patterns will remain valid in the future. However, due to the current climate change evidences this approach is no longer reliable. Therefore decision support tools, using physical modelling with policy definition, needs to incorporate the forecasts of climate change and its associated uncertainty.

As mentioned in [1] "Adaptation to climate change presents a complex methodological challenge. It calls for individuals to make decisions with potentially long-term consequences based on incomplete knowledge and uncertain information".

Several studies have investigated the effects of climate change on reservoirs [2]-[7], the majority of these predicting worsening reservoir performance and higher storage capacity requirements due to climatic change. Most of these studies use General Circulation Models (GCMs) as drivers for the future Climate Change (CC) inflow in the system modelled. Some authors [7], [8] used an ensemble of current and future climate to characterize the variability through a set of possible scenarios, that are then used as inflow for rainfall-runoff models.

In this paper we are proposing to characterize the inflow uncertainty through a stochastic generated ensemble that feeds a rainfall-runoff model. This is the entry for a reservoir model that incorporates policy derived management, controlling the outflows of the system. The method proposed incorporates a two state Markov Chain (MC) model supported by a "signature" of the monthly pattern both for the historical record and for the bias corrected CC GCM models. The parameters of the MC are used to generate a smooth transition from the historical to the climate impacted models.

Based on this methodology we, developed a decision support tool for reservoir management strategies. We use the historical data of the Cantareira reservoir, responsible for almost half of the water supply to São Paulo (Brazil's largest city). 
Historical records show that rainfall pattern of rainy summer, followed by dry winters, have seen more variation in the last 5 years than the previous 60 years recorded.

The Cantareira reservoir experienced a drought from 2014 to 2015 that resulted in the rationalization of the water supply. This reduction amounted up to $50 \%$ flow reductions for some millions in the city [9].

During the crisis continuous stakeholder meetings were necessary to define the management of the reservoir, as the previous legal indicators did not contemplate this level of stress in the system. Another impact was a new proposition of the legal limits imposed by the stakeholders on the reservoir management [10], which is also incorporated in our implementation in this paper (i.e. RAC2).

\section{DATASET AND METHODS}

We have used the public data available daily (from 2006 to 2016) for the Cantareira reservoir [11] as a lumped system.

A generic view of the system we will simulate is shown in Fig. 1.

The component "Rainfall" integrates the effective runoff and incorporates both historical data and GCM with CC forecasted impact, it is related to block "Data wrangling" and "stochastic simulation" as presented in more detail in Fig. 2.

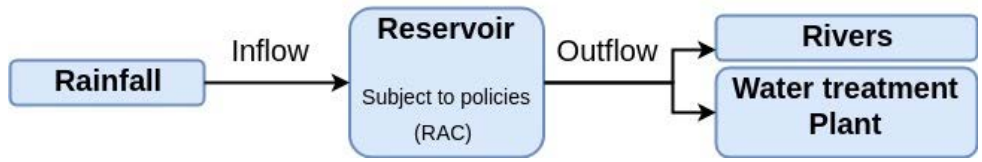

Figure 1: Schematic representation of the system.
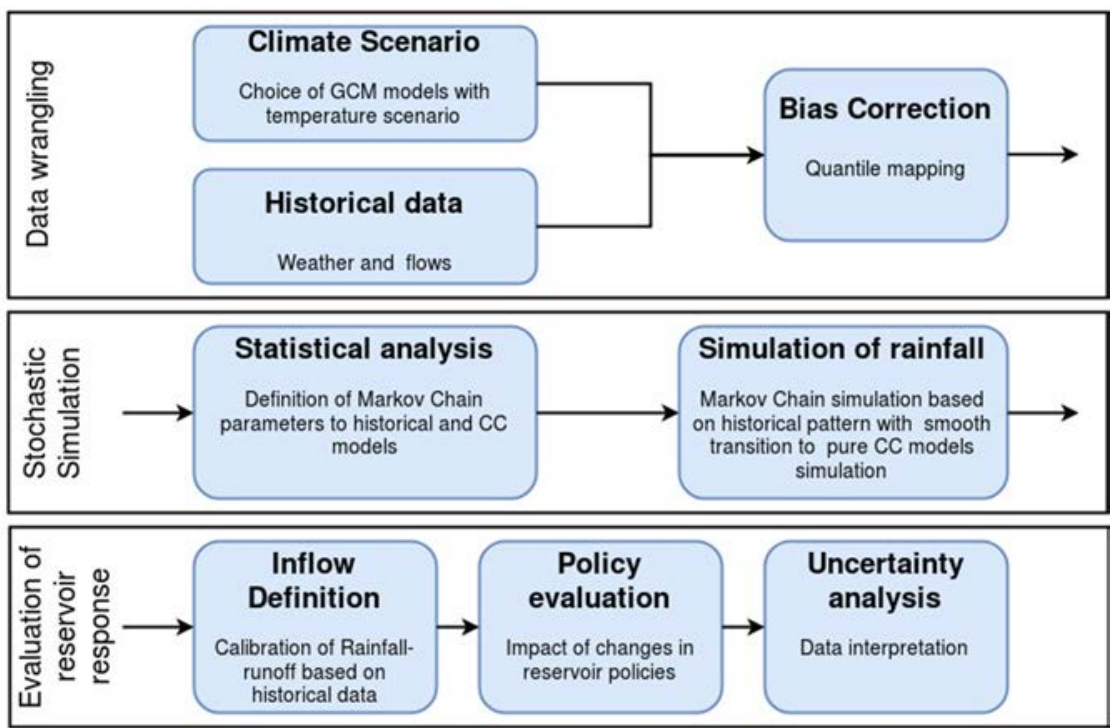

Figure 2: Flowchart defining the simulation steps described in the paragraphs ahead. We have chosen HADGEM and CNRM models [12]-[14] for evaluation of CC impact here. 


\subsection{Bias correction by Quantile Mapping}

As is well known, the biases present in the outputs from GCMs affect all aspects of the intensity range and must be corrected to provide the best input to the rainfall-runoff model [15], [16].

The bias correction method adopted in this study consists of an empirical Quantile Mapping (QM) [16], [17]. This method aims to define a quantile-specific Transfer Function (TF) determined by the difference between simulated and observed empirical cumulative distribution functions (ecdf), over a common historical period, in our case 10 years. In the literature alternative names refer to the same technique as: statistical downscaling, quintile mapping, histogram equalizing and rank matching.

The QM correction we used is defined as:

$$
\text { Pcor }_{m, d}=e c d f_{o b s, m}^{-1}\left(\operatorname{ecd} f_{\text {raw }, m}\left(P_{\text {raw }, m d}\right)\right) \text {, }
$$

- $\quad \operatorname{Pcor}_{m, d}$ : the corrected value of precipitation for month $m$ and day $d$;

- $e c d f^{-1}{ }_{o b s, m}$ is the inverse of ecdf (Empirical Cumulative Distribution Function) for the observation of the month $m$;

- $\quad P_{\text {raw,m } d}$ is the raw (biased) data for month $m$, and day $d$.

In eqn (1) a daily based TF is given by the mismatch between both observed and raw inverse empirical cumulative distribution functions (quantile functions), during the calibration period: $e c d f_{o b s, m}^{-1}$ and $e c d f_{\text {raw, } m}$.

Once the TF is determined, it is used in the rest of the simulation over the CC models.

The resulting QM correction for the CC models and the raw Cantareira is shown in Fig. 3.

It is interesting to notice that given the bias correction on the $c d f$ on a period of 10 years, there are changes both reducing or increasing locally the discrepancy with the historical data, but the integral of the rainfall during this period is conditioned to the historical measurements as shown in Fig. 4.

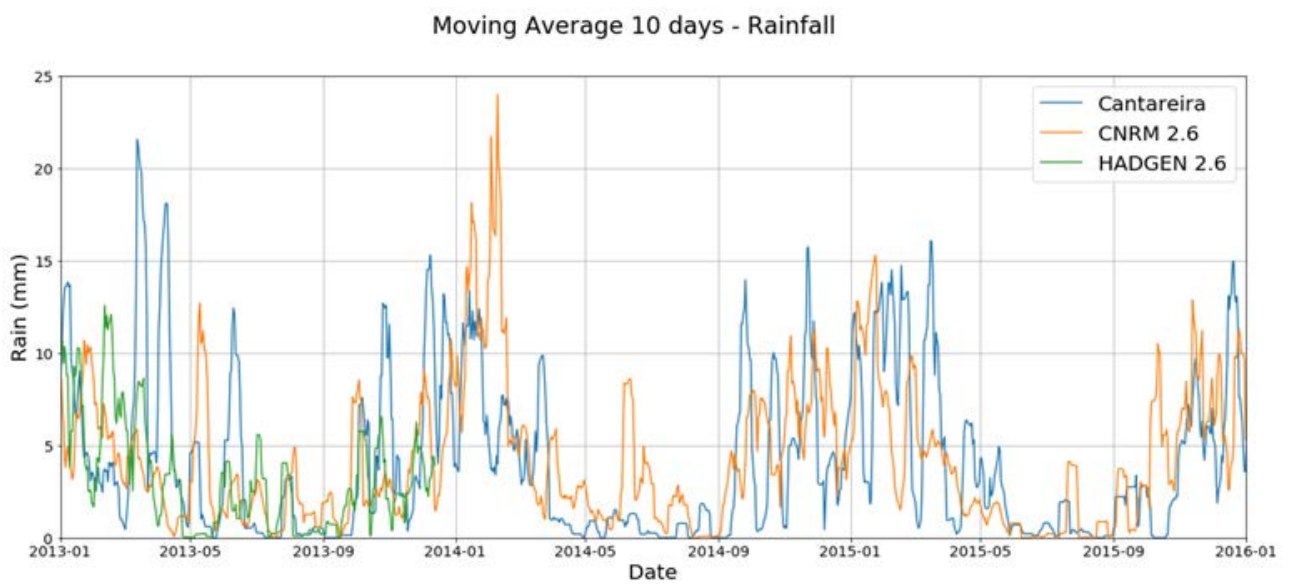

Figure 3: HADGEM (green) and CNRM (orange) rainfall moving average window of 10 days after QM correction, historical Cantareira rainfall in blue. 


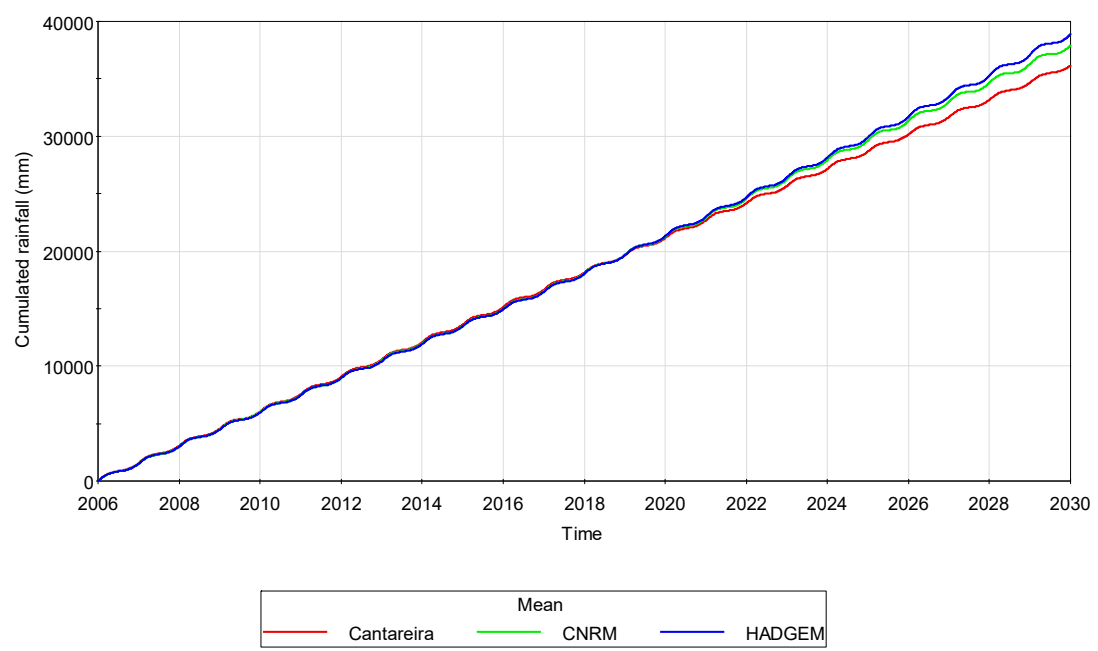

Figure 4: Integral of rainfall with Historical data (red), HADGEN after QM (blue), CNRM (green). The lines superimpose until 2016, the calibration period.

\subsection{Markov Chain model to stochastic rainfall simulation}

Markov Chain (MC) models have been used to generate ensembles of similar temporal patterns. [7], [18] show successful applications to simulate rainfall.

The MC used here is based on a statistical parameterisation of the historical series by the probability of being in two possible daily states: "rain" or "not rain", while the intensity of the rain event was adjusted by an Exponential Distribution.

The MC method is based on a sequence of random variables $\left\{x_{t}\right\}$ :

$$
P\left(x_{t}=i_{t} \mid x_{0}=i_{0}, x_{1}=i_{1}, \ldots, x_{t-1}=i_{t-1}\right)=P\left(x_{t}=i_{t} \mid x_{t-1}=i_{t-1}\right) .
$$

The amount of rainfall can be modelled as a sequence of random variables:

$$
\left\{z_{t}\right\}, z_{t}=x_{t} y_{t},
$$

where $\left\{x_{t}\right\}$ is a sequence of discrete random variables that form a Markov Chain and $\left\{y_{t}\right\}$ a sequence of continuous random variables.

- $\left\{x_{t}\right\}$ defines whether or not there was rain on day $t$ (i.e. $x_{t}=1$, representing a rainy day and $x_{t}=0$ representing a dry day),

- $\left\{y_{t}\right\}$ sets the amount of rain (if any) on day $t$.

The transition probabilities are defined according with the day and the month, as follows:

$$
p_{i, j, m}=P\left(x_{t}=j \mid x_{t-1}=i\right), i, j \in\{0,1\},
$$

- $\quad m$ defines the month of the day $t$.

- $\quad i$ and $j$ are the two possible states (i.e. rain (1) or not rain (0)) represented by this MC process.

From this setup, we can generate stochastic series of rain that reproduce the historical patterns of a region defined from Monte-Carlo simulations. 
For this, the approximate form of $p_{i, j, m}$ is:

$$
\begin{aligned}
p_{0,1, m}=\frac{a_{0,1, m}}{a_{1,0, m}+a_{0,0, m}} \quad p_{1,1, m} & =\frac{a_{1,1, m}}{a_{0,1, m}+a_{1,1, m}}, \\
p_{0,1, m}+p_{0,0, m}=p_{1,0, m}+p_{1,1, m} & =1,
\end{aligned}
$$

where $a_{i, j, m}$ represents the historical frequency at which the transition $\left\{x_{t}=j, x_{t-1}=i\right\}$ occurred for the month $m$. The gamma, log-normal or exponential functions can be used approximate rainfall distributions $\left\{y_{t, m}\right\}$ for each month $m$, and $\overline{y_{m}}$ represents the average rainfall of rainy days. The exponential function provides a good fit to historical data.

Fig. 5 shows the MC parameters (P01 and P11) obtained after the QM bias correction for $\mathrm{CC}$ models scenario $2.6^{\circ} \mathrm{C}$ temperature increase.

\subsection{Simulating a smooth transition from historical to climate impacted scenario}

We consider that we are probably in a transition period between the historical and the CC impacted climate. The use of the MC technique allows us to explore a new approach, by simulating this transition of rainfall patterns smoothly.

Performing a gradual scenario change simulation consists of applying an intermediate transition parameter from the historical to any of the $\mathrm{CC}$ bias corrected scenarios. A simple way to define these intermediate parameters is to linearly interpolate the transition parameters $\left(p_{0,1, m}, p_{1,1, m}\right.$ e $\left.\overline{y_{m}}\right)$ over the years. Fig. 6 is an example of a transition occurring during a 10 years span.

Fig. 7 Exhibits the changes in the average precipitation between the $\mathrm{CC}$ models and the historical patterns. An increase in the intensity of dry and wet periods can be observed when considering the CC models in the last years simulated.

\subsection{Runoff modelling}

To translate the forecasted precipitation from climate change models into inflow for the system, we calibrated a runoff watershed model based on the Australian Water Based Model $(\mathrm{AWBM})[20]$ that is widely used in tropical regions.

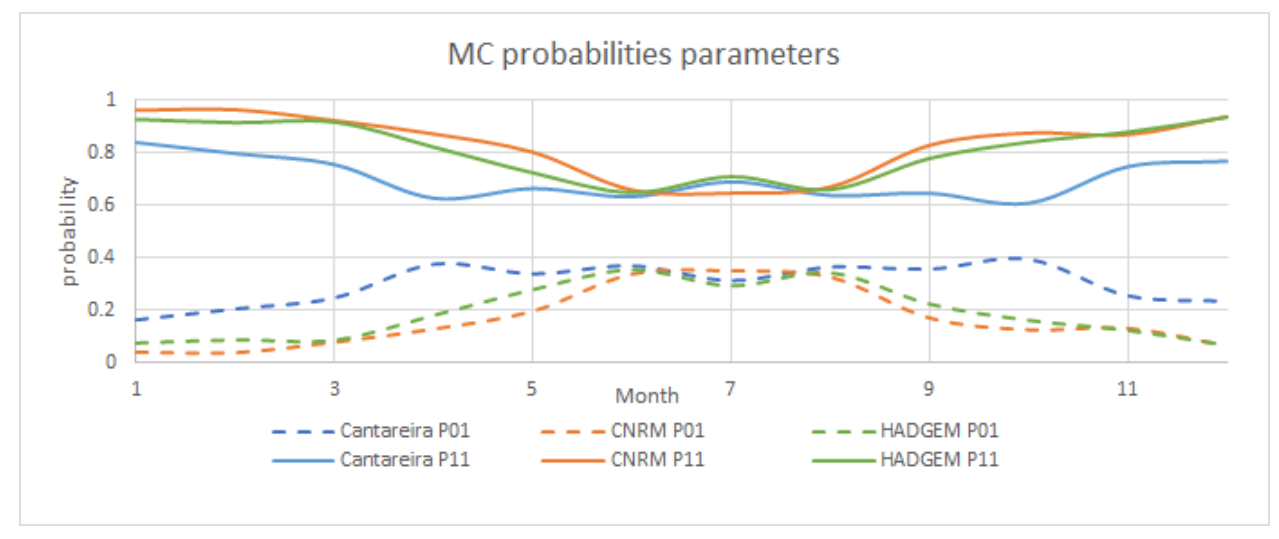

Figure 5: $\quad$ MC probabilities P01 in dashed (Probability of do not rain (0) given it rained (1) the day before) and P11 full line (Probability of rain (1) given it rained (1) the day before) for the Historical (blue), CNRM (orange), HADGEM (green). 




Figure 6: Example of transition probability from Cantareira pattern (dark blue) to CC impacted (in colour for each year) until show pure CC at year 10 (light blue).



Figure 7: Monthly average rainfall from historical pattern (red), CNRM (green) HADGEM (blue). Notice that after 2016 the CC models tends to increase variability in relation to historical simulated.

Given the drought period had long dry periods in relation to historical data and due to difficulties in attaining a reasonable calibration for the dry period without compromising the wet period, we incorporated a groundwater element to the model (see Fig. 8). The groundwater store becomes active to maintain a minimum flow. The activation occurs only when the inflow is lower than a threshold and it facilitates the calibration with the specific focus on drought periods.

We have adopted a simplified Evapotranspiration based on historical monthly pattern of the region. Fig. 9 shows the resulting calibrated model.

With the runoff model calibrated we used both the stochastic model of the Cantareira and the $\mathrm{CC}$ simulations as inputs to provide the inflow to the reservoir model management. 


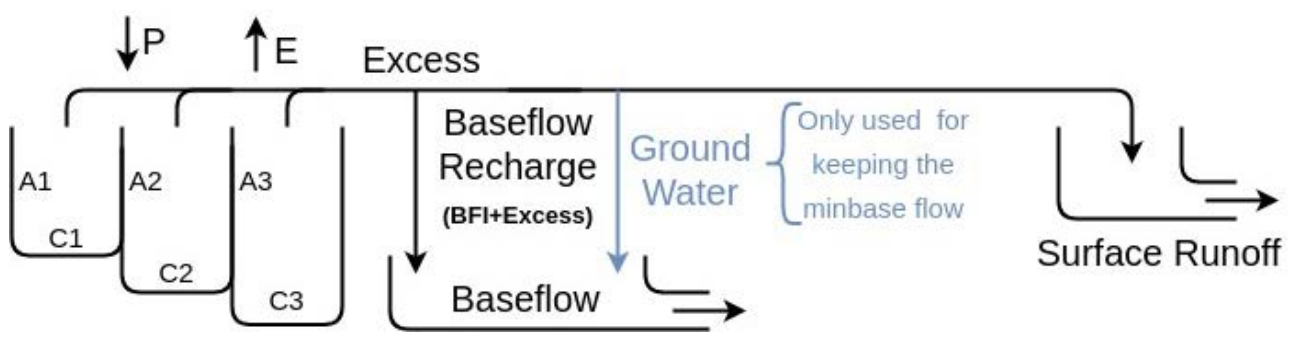

Figure 8: AWBM components and the new parameter used to define the base flow in drought periods, groundwater (blue). Here P: precipitation, E: evapotranspiration, BFI: Base Flow index, $\mathrm{C}$ and $\mathrm{A}$ are parameters representing surface area fraction and water storage capacity, with i: 1,2,3 representing three zones. (Source: adapted from [19].)

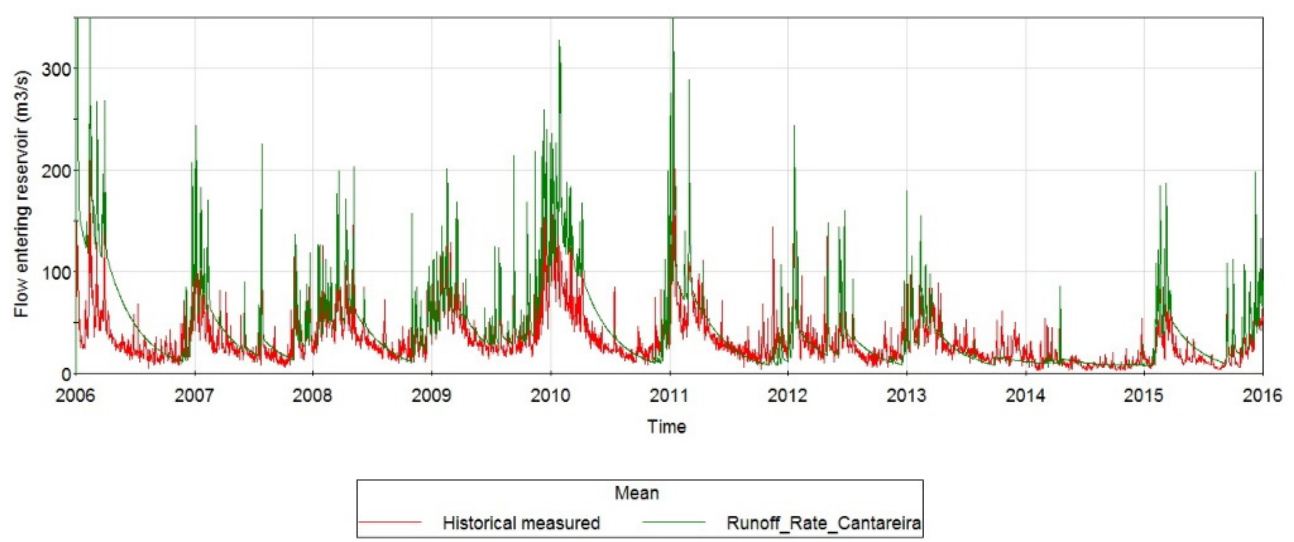

Figure 9: Calibration of the runoff model, red is historical data, green is modelled runoff from historical rainfall on daily data. Notice the driest period after mid-2013.

\subsection{Management of the reservoir operation}

The reservoir has storage capacity of $978 \mathrm{~mm}^{3}$ with drainage surface of $2300 \mathrm{~km}^{2}$, it is designed to feed a water treatment plant (WTP) and must maintain minimum flow in rivers for two different watersheds.

The system has a legally defined management process controlled by a Risk Aversion Curve (RAC) [10]. The RAC indicated the maximum allowed withdrawal rate for the WTP and the minimum flow for three rivers downstream. Fig. 10(a) show the "seasonal policy" system used until 2016 (RAC1) and Fig. 10(b) the newly adopted policy (RAC2).

The WTP has a pumping system able to treat $33 \mathrm{~m}^{3} / \mathrm{s}$; while the minimum legal outflow to the rivers ranges from $3 \mathrm{~m}^{3} / \mathrm{s}$ up to $8 \mathrm{~m}^{3} / \mathrm{s}$ according with the reservoir level and the priority defined by the RAC (not shown here). 


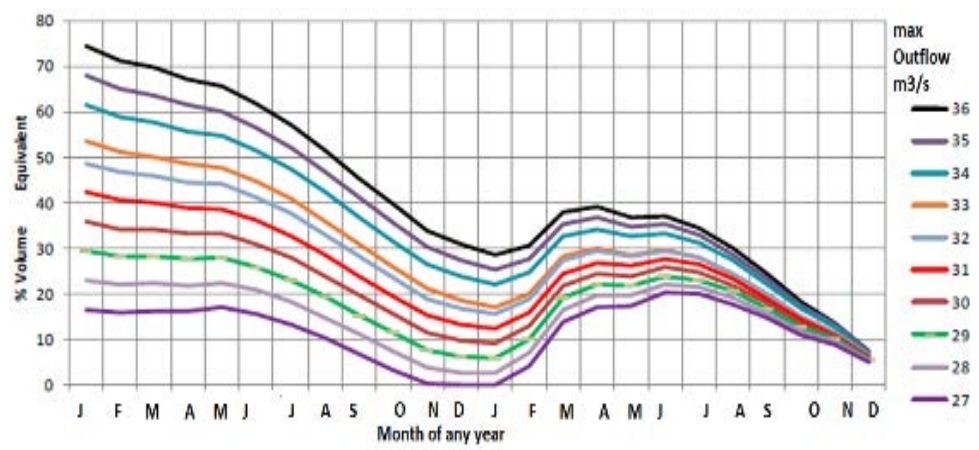

(a)



(b)

Figure 10: Risk Aversion Curve (RAC) indicate outflows imposed maximum (for WTP) and minimum (for rivers). RAC1 shown in (a) with maximum outflow related to reservoir level in a given month; and RAC2 shown in (b) with limit of outflow for WTP according with percentage of the storage without relation with month. (Source: adapted from [10].)

\section{STOCHASTIC SIMULATION OF CLIMATE CHANGE IMPACT IN THE RESERVOIR}

For the following plots, we show the real QM corrected data of Cantareira for the period before August 2016. After this date, all models incorporate the MC simulation as explained previously and, as such, can be analysed as a stochastic process with probabilities of reaching a certain level.

\subsection{Simulation of policy impact based in historical pattern}

For comparing the two policies, we used 100 Cantareira MC simulations and obtained the probabilities of reaching a reservoir levels shown in Fig. 11 (RAC1 in 11(a), RAC2 in 11(b)). It shows that under RAC2 there is a reduction in volumes stored as there is an increase in probabilities related to lower levels.

The impact of these levels in the effective allowed outflow feeding the Water Treatment Plant is shown in Fig. 12. Given that the normal operation for the city requires about $31 \mathrm{~m}^{3} / \mathrm{s}$, this simulation shows for a long time a risk between $5 \%$ and $25 \%$ of a reduction in availability up to half of the normal operation necessity. 

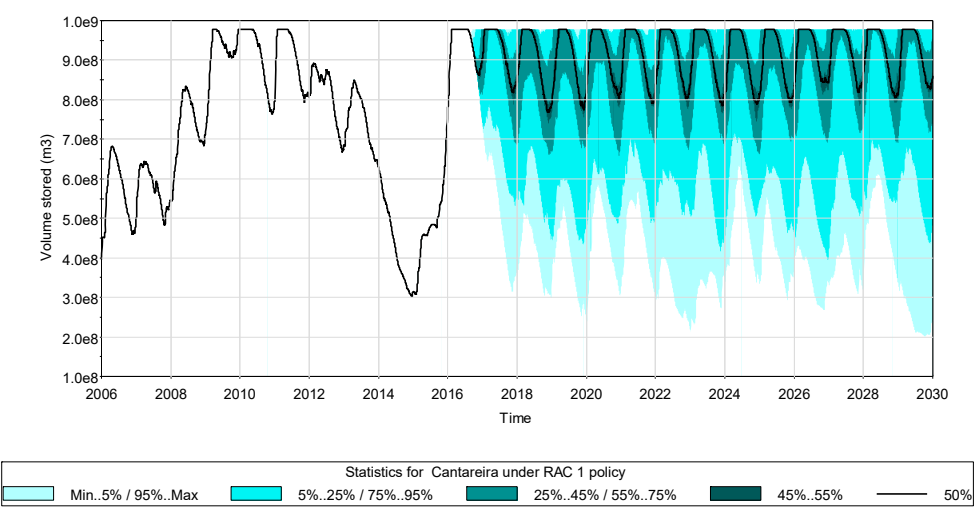

(a)



Min..5\%/ $95 \% . \operatorname{Max}$

Statistics for Cantareira under RAC2 policy

(b)

Figure 11: Volumes stored simulated with MC Cantareira data, in (a) under policy RAC1; and (b) under RAC2. The latter provides lower levels in reservoir.


Figure 12: Flow allowed to be pumped to WTP, simulated under RAC1 policy, it show an increased risk with time. 


\subsection{Simulation of climate change impact}

Comparing the two CC models under RAC1 (see Fig. 13) we can clearly see they both show an increase in inflow and higher reservoir levels than the historical Cantareira pattern (comparing to Fig. 11(a)).

These models also indicate that there is an increase of reservoir overflow probability when the CC models are used.


(a)

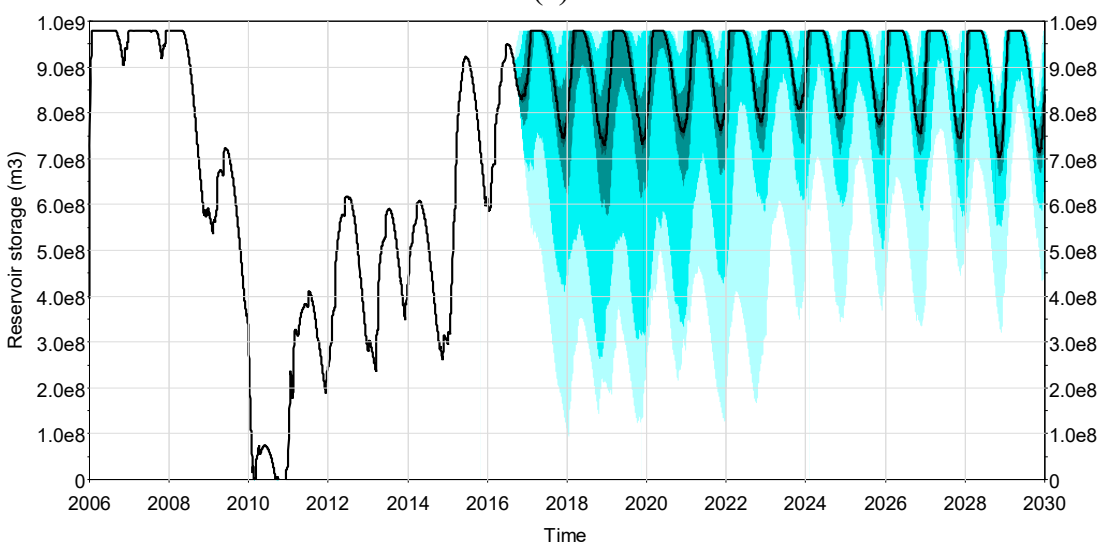

\begin{tabular}{ll} 
& \multicolumn{2}{c}{ Statistics for CNRM under CAR1 } \\
Min..5\% / 95\%..Max & $5 \% . .25 \% / 75 \% . .95 \%$ \\
$45 \% .55 \%$ & $50 \%$
\end{tabular}

$25 \% . .45 \% / 55 \% . .75 \%$

(b)

Figure 13: Volumes stored, simulated under RAC1 policy, with CC model HADGEM (a) and CNRM (b). This last indicates higher fluctuations to lower levels in reservoir than the previous and both show an increase in inflow with time as reservoir levels tends to be higher with time. 


\section{CONCLUSION}

We have developed a methodology utilizing a stochastic generator of rainfall. It is used for incorporating $\mathrm{CC}$ signals and reservoir policy evaluation.

The CC simulations were obtained from two bias corrected CGM models (HADGEM and CNRM).

A stochastic simulation using Markov Chain was used to generate a pattern like the historical records and a new proposition, of a smooth transition from this pattern to the pattern obtained from the bias corrected CC models detailed.

The stochastic rainfall prediction was used as inflow in a calibrated runoff model to provide the effective recharge to the reservoir. The reservoir levels were simulated based on two policies, and the amount of water available for the water treatment plant calculated.

The analysis of these simulations indicates that using only historical patterns the new policy (RAC2) would provide lower levels in the reservoir. The impact of this inflows for the allowed withdraw feeding the WTP show an increase in probability of reduction in availability up to half of the normal operation in some months (simulation done until 2030).

The CC simulations show that both models (considering $2.6^{\circ} \mathrm{C}$ scenario) reduce the risk of low levels in the reservoir.

\section{REFERENCES}

[1] Climate-ADAPT, Sharing adaptation information across Europe, Online. http://climate-adapt.eea.europa.eu/knowledge/tools/uncertainty-guidance/topic1. Accessed on: Apr. 2018.

[2] Fowler, H.J., Kilsby, C.G. \& O'Connell, P.E., Modeling the impacts of climatic change and variability on the reliability, resilience, and vulnerability of a water resource system. Water Resources Research, 39(8), 2003.

[3] Nawaz, N.R. \& Adeloye, A.J., Monte Carlo assessment of sampling uncertainty of climate change impacts on water resources yield in Yorkshire, England. Climatic Change, 78(2-4), pp. 257-292, 2006.

[4] Burn, D.H. \& Simonovic, S.P., Sensitivity of reservoir operation performance to climatic change. Water Resources Management, 10(6), pp. 463-478, 1996.

[5] Li, L. et al., A fuzzy analytic hierarchy process (FAHP) approach to eco-environmental vulnerability assessment for the Danjiangkou reservoir area, China. Ecological Modelling, 220(23), pp. 3439-3447, 2009.

[6] Raje, D. \& Mujumdar, P.P., Reservoir performance under uncertainty in hydrologic impacts of climate change. Advances in Water Resources, 33(3), pp. 312-326, 2010.

[7] Arshad H., Thesis. Department of Civil Engineering and Applied Mechanics, McGill University: Montreal, 2008.

[8] Peel, M.C. \& Blöschl, G., Hydrological modelling in a changing world. Progress in Physical Geography, 35(2), pp. 249-261, 2011.

[9] Crise hídrica no estado de São Paulo em 2014-2016, Online. https://pt.wikipedia.org/wiki/Crise_h\%C3\%ADdrica_no_estado_de_S\%C3\%A3o_ Paulo_em_2014\%E2\%80\%932016. Accessed on: Apr. 2018.

[10] ANA 2014, Renovação da Outorga do Sistema Cantareira, Online. www3.ana.gov.br/portal/ANA/sala-de-situacao/sistema-cantareira/renovacao-daoutorga-do-sistema-cantareira. Accessed on: Apr. 2018.

[11] ANA 2018, Sala de situação, Online. www3.ana.gov.br/portal/ANA/sala-de-situacao/ sistema-cantareira. Accessed on Apr. 2016.

[12] The HadGEM2 family of Met Office Unified Model climate configurations. Geosci. Model Dev., 4, pp. 723-757. DOI: 10.5194/gmd-4-723-2011. 
[13] Collins, W.J. et al., Development and evaluation of an Earth-System modelHadGEM2. Geosci. Model Dev., 4, pp. 1051-1075, 2011.

[14] Voldoire, A. et al., The CNRM-CM5.1 global climate model: Description and basic evaluation. Climate Dynamics, 40, p. 2091, 2013. DOI: 10.1007/s00382-011-1259-y.

[15] Baigorria, G.A. et al., Assessing uncertainties in crop model simulations using daily bias-corrected regional circulation model outputs. Climate Research, 34(3), pp. 211222, 2007.

[16] Piani, C., Haerter, J.O. \& Coppola, E., Statistical bias correction for daily precipitation in regional climate models over Europe. Theoretical and Applied Climatology, 99(12), pp. 187-192, 2010.

[17] Panofsky, H.A. \& Brier, G.W., Some Applications of Statistics to Meteorology. Earth and Mineral Sciences Continuing Education, College of Earth and Mineral Sciences, 1968.

[18] Sang-Yoon, H., Stochastic Modeling of Rainfall Processes: A Markov Chain - Mixed Exponential Model for Rainfalls in Different Climatic Conditions. Thesis, McGill University, 2001.

[19] Boughton, W., The Australian water balance model. Environmental Modelling \& Software, 19(10), pp. 943-956, 2004. 